\title{
Dynamic Coupling Analysis of Vehicle-Bridge System for Long-Span Suspension Bridge Based on Backpropagation Neural Network Method
}

\author{
Zuolong Luo $\mathbb{D}^{1},{ }^{1}$ Xiaobo Zheng $\mathbb{D},^{2}$ Haoyun Yuan $\mathbb{D}^{3},{ }^{3}$ and Xirong Niu ${ }^{1}$ \\ ${ }^{1}$ Department of Civil Engineering, Shanxi University, Taiyuan 030000, China \\ ${ }^{2}$ Key Laboratory of Transport Industry of Bridge Detection Reinforcement Technology, Chang'an University, Xi'an 710064, China \\ ${ }^{3}$ Department of Bridge Engineering, Chang'an University, Xi'an 710064, China
}

Correspondence should be addressed to Xiaobo Zheng; zhengshi.yan@163.com

Received 18 January 2020; Revised 7 September 2020; Accepted 15 September 2020; Published 25 September 2020

Academic Editor: Xueping Fan

Copyright (c) 2020 Zuolong Luo et al. This is an open access article distributed under the Creative Commons Attribution License, which permits unrestricted use, distribution, and reproduction in any medium, provided the original work is properly cited.

As the suspension bridge structures become more flexible and the forms of the vehicle load become more diverse, the dynamic coupling problem of the vehicle-bridge system has become gradually prominent in long-span suspension bridges, resulting in an increase in accuracy and efficiency requirements for dynamic coupling analysis of the vehicle-bridge system. Conventional method such as finite element method (FEM) for dynamic coupling analysis of vehicle-bridge system often requires separate iteration of vehicle system and bridge system, and the contact and coupling interactions between them are used as the link for convergence inspection, which is too computationally intensive and time-consuming. In addition, the dynamic response of the vehicle-bridge coupling system obtained by FEM cannot be expressed explicitly, which is not convenient for engineering application. To overcome these drawbacks mentioned above, the backpropagation (BP) neural network technology is proposed to the dynamic coupling analysis of the vehicle-bridge system of long-span suspension bridges. Firstly, the BP neural network was used to approximate the dynamic response of the suspension bridge in the vehicle-bridge coupling system, and the complex finite element analysis results were thus explicitly displayed in the form of a mathematical analytical expression. And then the dynamic response of the suspension bridge under vehicle load was obtained by using a dynamic explicit analysis method. It is shown through a numerical example that, compared with FEM, the proposed method is much more economical to achieve reasonable accuracy when dealing with the dynamic coupling problem of the vehicle-bridge system. Finally, an engineering case involving a detailed finite element model of a long-span suspension bridge with a main span of $1688 \mathrm{~m}$ is presented to demonstrate the applicability and efficiency under the premise of ensuring the approximation accuracy, which indicates that the proposed method provides a new approach for dynamic coupling analysis of the vehicle-bridge system of long-span suspension bridges.

\section{Introduction}

With the development of bridge engineering in the direction of long span, lightweight, and more flexibility, as well as improvement of vehicle load forms, wheel weight, and driving speed, the dynamic interaction between vehicle and bridge has been increasingly emphasized. As a dynamic load, vehicle load will cause bridge vibration and dynamic response, which is not only larger than the static load but also related to many factors such as dynamic characteristics of vehicle and bridge, driving speed, and road roughness [1-3].
The dynamic coupling effect of the vehicle-bridge system is complicated, such as affecting bridge safety and driving comfort and causing fatigue and durability problems. Therefore, it is essential to accurately analyze the dynamic coupling response of the vehicle-bridge system. Jiang et al. [4] decomposed the time-varying linear dynamic system into superposition of time-varying linear dynamic system and time-invariant linear dynamic system and established the state-space method based on state-space theory for dynamic coupling analysis of the vehicle-bridge system using the finite element method and studied the dynamic 
coupling response of the vehicle-bridge system of suspension bridge under vehicle load. Li et al. [5] derived both vibration equations of vehicle and bridge by using the virtual work principle and finite element method and then studied the dynamic response of a single-tower self-anchored suspension bridge under different road roughness, vehicle speed, and bridge damping. Ding et al. [6] deduced the motion equation of dynamic coupling response of vehiclebridge system based on D'Alembert's principle and deformation compatibility relations using a single moving massspring-damping model to simulate the vehicle and discussed the influence of single moving vehicle load on the vibration response of double-chain suspension bridge in conditions of vehicle driving with the center of the bridge and eccentric driving considering geometric nonlinearity and bridge deck roughness. Further research was carried out by Li et al. [7], who established a relatively complete dynamic model of vehicle-bridge coupling system on the basis of dynamic coupling theory of vehicle-bridge system theory, and the dynamic performance of a suspension bridge with a main span of $1120 \mathrm{~m}$, track, and vehicle under the load of ICE3 train at a speed of $160-300 \mathrm{~km} / \mathrm{h}$ as well as the dynamic response of bridge and vehicle under different main cable forces and the cable forces was also studied. Liu et al. [8] proposed a typical three-dimensional multiaxle vehicle model and generated three different road roughness models evaluated as excellent, good, and normal using power spectral density, and the random traffic flow of 16 kinds of vehicle types was simulated. In addition, a finite element model of stiffening beam was established to study the vehicle vibration effect, and vehicle parameters, road roughness, driving directions, and driving speed were also calculated. In order to investigate the dynamic response of railway suspension bridge in the wind field under the trainload closer to reality, an improved sequential analysis model of windvehicle-bridge coupling system considering the nonlinear effect of large displacement and little strain was presented by Wang [9] whose research focused on the vibration response of railway suspension bridge under the trainload and wind load, along with the producing mechanism and changing rule of extreme response values.

Summing up the above studies, we can find that some progress pointing at the basic theory and method as well as the model sensitivity analysis of the dynamic coupling response of vehicle-bridge system has been made in decades; however, there are mainly two issues remaining to study. Firstly, conventional methods, such as the finite element method, for dynamic coupling analysis of vehicle-bridge system often require separate iteration of the vehicle system and bridge system, and the contact and coupling interactions between the two systems are used as the link for convergence inspection, which is too computationally intensive and timeconsuming. Secondly, the bridge response obtained by conventional method for dynamic coupling analysis of the vehicle-bridge system cannot be expressed explicitly, which is not convenient for engineering application. To the best of the authors' knowledge, the backpropagation (BP) neural network is usually used in the artificial intelligence field, which simulates the mode of human brain receiving and processing information, adopts a distributed processing mechanism, composes a complex network through different connection ways of basic neurons, and forms a powerful machine-learning algorithm for functions implementation such as classification, clustering, fitting, prediction, and compression [10-16]. BP neural network is a self-adaption nonlinear dynamic system which is composed of substantial neurons, each of which has a relatively simple structure and function, but the system consisting of a large number of neurons after combination will have a very sophisticated behavior. In addition, when the topological structure of BP neural network is set reasonably, the transfer function has a nonlinear characteristic, and the BP neural network has obtained reasonable connection authority and threshold after appropriate training, the BP neural network can approach any mapping relation essentially. It is shown that $\mathrm{BP}$ neural network has strong learning ability and generalization ability, which makes BP neural network become an effective tool for converting implicit expression to explicit one [17-22].

Therefore, based on the iterative algorithm of vehiclebridge separation, this paper proposed a new approach for dynamic coupling analysis of vehicle-bridge system analysis, in which the BP neural network technology was introduced to solve the issue that it is difficult to express the formula of the bridge response explicitly in dynamic coupling analysis of the vehicle-bridge system. Besides, the computational workload will have a significant decrease in the precondition of meeting the accuracy requirements for actual engineering analysis when the BP neural network technology is used to approximate the bridge response under the dynamic coupling effect of the vehicle-bridge system effect, so as to obtain the explicit expression of the dynamic response and to predict the bridge dynamic response of other vehicle-bridge coupling types.

\section{Proposed Method}

2.1. Dynamic Coupling Theory of Vehicle-Bridge System. The dynamic coupling analysis model of the vehicle-bridge system, which was shown in Figure 1, consists of vehicle dynamic model and bridge dynamic model, which interact on each other by the connection between wheel and bridge deck and can be established according to the rigid-body dynamic method and finite element method, respectively. In terms of a bridge structure, its final dynamic equilibrium equation always has the following form:

$$
M_{b} \ddot{y}_{b}+C_{b} \dot{y}_{b}+K_{b} y_{b}=F,
$$

where $M_{b}, C_{b}$, and $K_{b}$ are the mass stiffness matrix of the bridge, damping stiffness matrix of the bridge, and stiffness matrix of the bridge, respectively; $\ddot{y}_{b}, \dot{y}_{b}$, and $y_{b}$, respectively, represent the acceleration vector of bridge discrete nodes, velocity vector of bridge discrete nodes, and displacement vector of bridge discrete nodes; $F$ is the vehicle dynamic load on the bridge structure, which can be obtained through the iterative computation of the vehicle system dynamic model. 


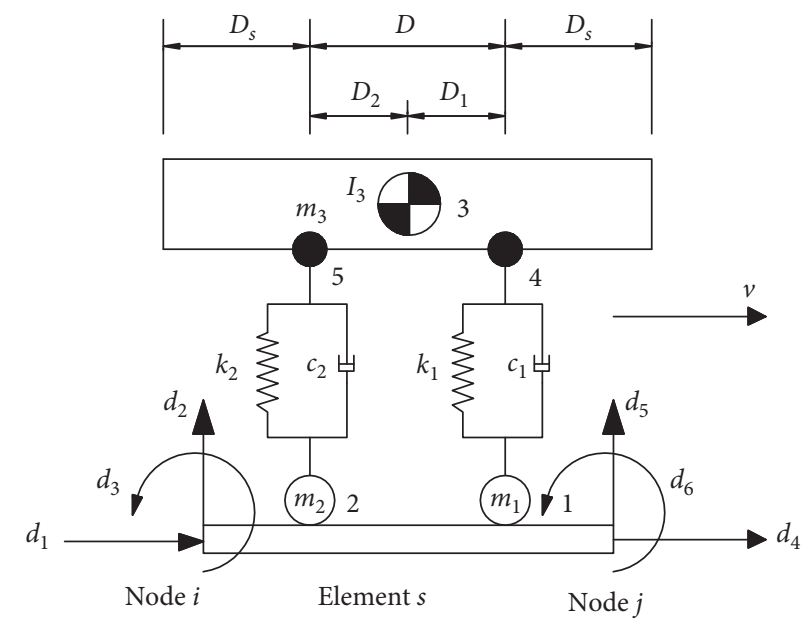

Figure 1: Vehicle-bridge system.

Based on the above vehicle-bridge coupling vibration equation, the general motion equation of vehicle-bridge interaction is obtained as follows:

$$
\left\{\left[\begin{array}{cc}
M_{b} & 0 \\
0 & M_{u n}
\end{array}\right]+M_{\mathrm{vib}}\right\}\left(\begin{array}{c}
\ddot{d} \\
\ddot{y}_{u}
\end{array}\right)+\left\{\left[\begin{array}{cc}
C_{b} & 0 \\
0 & C_{u n}
\end{array}\right]+C_{\mathrm{vib}}\right\}\left(\begin{array}{c}
\dot{d} \\
\dot{y}_{u}
\end{array}\right)+\left\{\left[\begin{array}{cc}
K_{b} & 0 \\
0 & K_{u n}
\end{array}\right]+K_{\mathrm{vib}}\right\}\left(\begin{array}{l}
d \\
y_{u}
\end{array}\right)=\left(\begin{array}{l}
F_{b} \\
F_{c u}
\end{array}\right),
$$

where the corner marker vib means the time-varying system. The system with variable mass, stiffness, and damping can be solved by the system simulation module of the finite element analysis, and the displacement response of each point in the physical coordinate system can be obtained directly.

The above formula can be expressed as

$$
\left\{M+M_{\mathrm{vib}}\right\}\left(\begin{array}{c}
\ddot{d} \\
\ddot{y}_{u}
\end{array}\right)+\left\{C+C_{\mathrm{vib}}\right\}\left(\begin{array}{c}
\dot{d} \\
\dot{y}_{u}
\end{array}\right)+\left\{K+K_{\mathrm{vib}}\right\}\left(\begin{array}{c}
d \\
y_{u}
\end{array}\right)=F,
$$

where the matrix with vib was time-variable and the modal matrix of the whole system also changes with time. The modal matrix is $\Phi$, and equation (3) can be written as

$$
M_{p} \ddot{q}+C_{p} \dot{q}+K_{p} q=\Phi^{\mathrm{T}} F
$$

where

$$
\begin{aligned}
\left(\mathrm{d} y_{u}\right)^{T} & =\Phi q \\
M_{p} & =\Phi^{T} M \Phi+\Phi^{T} M_{\mathrm{vib}} \Phi \\
C_{p} & =\Phi^{T} C \Phi+\Phi^{T} C_{\mathrm{vib}} \Phi \\
K_{p} & =\Phi^{T} K \Phi+\Phi^{T} K_{\mathrm{vib}} \Phi .
\end{aligned}
$$

The system matrixes $M, C$, and $K$ consist of two parts which are the system of bridge and the body part of vehicle, respectively. The modal matrix $\Phi$ is also divided into modal matrix of bridge and moment of vehicle body part. For the complex model, the modal matrix of the bridge part can pass through the ANSYS software and the car body models can be obtained directly.

As for the vehicle system, which is customarily simulated as a spring, damping and mass system with multiple degrees of freedom, has two frequently used vehicle type: two-axle vehicle and three-axle vehicles, respectively. So, this paper mainly discussed the two vehicles model composed of mass and linear spring-damping system, in which the vehicle body possesses three degrees of freedom: two translational and one rotational, and each wheel has one vertical vibration degree of freedom. Therefore, the two-axle vehicle is 7 degrees of freedom system, while the three-axle vehicle is 9 degrees of freedom system.

In order to establish the motion equation of vehicle system, the influence of road surface roughness and bridge deck displacement should be considered, and the coordination equation is then established based on the condition of close contact between wheel and bridge deck. The dynamic analysis model of long-span suspension bridge is established by using the finite element software ANSYS, and its spatial free vibration characteristics are calculated. A three-dimensional vehicle model is established by using the multibody dynamics system. Finally, the spatial coupling vibration response of vehicle passing through the long-span suspension bridge at different speeds is calculated by using the joint simulation technology based on multibody system dynamics and finite element method.

It can be seen that the traditional finite element method is very complicated to simulate the coupling vibration of vehicle and bridge. Particularly, when a large number of simulation calculations are needed or the finite element 
method is used repeatedly for iterative analysis, the disadvantages of the finite element method are exposed. Therefore, it is necessary to find an accurate, efficient, and time-saving method to replace the finite element calculation. Neural network simulation has great advantages in this respect. The BP neural network has a high degree of nonlinear fitting characteristics and can save a lot of calculation time, so it has great advantages in engineering application.

2.2. BP Neural Network. This section will focus on the introduction of BP neural network technology into the dynamic coupling analysis of the vehicle-bridge system of longspan suspension bridge using BP neural network, in which the basic operation function is BP neuron, as a specific means, and the general model of BP neuron is shown in Figure 2.

The BP neural network is generally a multilayer BP neural network, which consists of multiple neurons, forming a distributed information learning and processing mechanism, and a typical architecture of a three-layer BP neural network composed of input layer, processing layer, and output layer is the most extensively BP neural network, whose operation principle is shown in Figure 3.

The BP network can learn and store a large number of mapping relationships of input/output modes without revealing the mathematical equations describing the mapping relationship, which can be expressed as equation (6), and the learning rule of BP network is to use the steepest descent algorithm to continuously adjust the weights and thresholds of the network through backpropagation, so as to minimize the sum of network squared errors:

$$
M: R^{k} \longrightarrow R^{d}, y=F(x)
$$

On the basis of the Kolmogorov continuity theorem, any continuous function can be approximated using a threelayer feed-forward BP network, and the mapping relationship $M$ of BP network can approach the function relationship $F$ which is hidden between $y$ and $x$. The approximation accuracy depends on the network structure and algorithm. Take a three-layer BP network with $k$ input nodes, $l$ hidden nodes, and $d$ output nodes as an example and assume that the input variable of the network is $x^{1}$; the values of the processing layer and output layer can be written, respectively, as equations (7) and (8):

$$
\begin{aligned}
& y_{m}^{2}=f^{2}\left(\sum_{i=1}^{k} \omega_{i m}^{1,2} x_{i}^{1}+\theta_{m}^{2}\right) ; m=1,2, \ldots, l, \\
& y_{n}^{3}=f^{3}\left(\sum_{m=1}^{l} \omega_{m n}^{2,3} y_{m}^{2}+\theta_{n}^{3}\right) ; n=1,2, \ldots d,
\end{aligned}
$$

where $x_{m}^{i}$ and $y_{m}^{i}$ represent input and output variables in the $m$ th node of the $i$ th layer, respectively; $\omega_{m n}^{i, j}$ refers to the connection weight between the $m$ th node of the $i$ th layer and the $n$th node of the $j$ th layer; $\theta_{m}^{i}$ is the threshold value in the

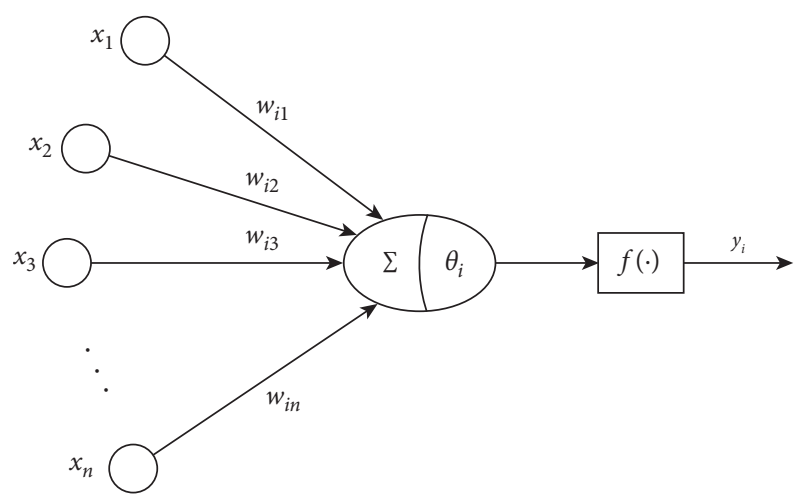

FIGURE 2: General model of BP neuron.

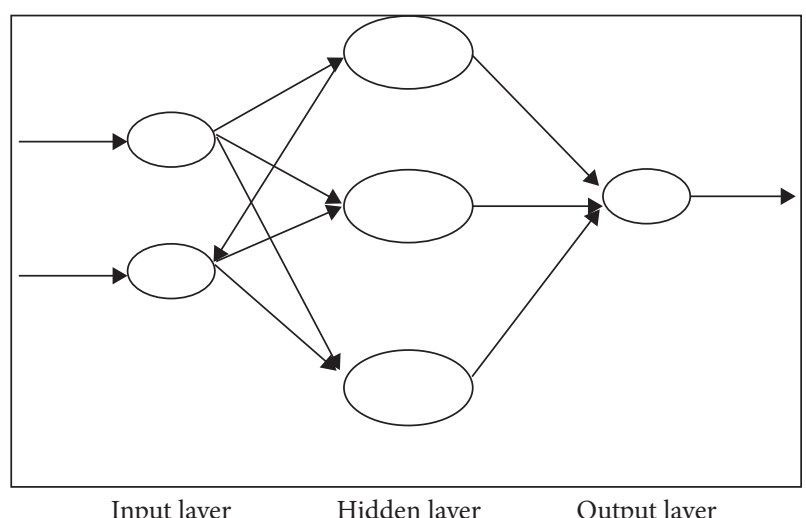

FIgURE 3: Schematic diagram of the three-layer BP neural network structure.

$m$ th node of the $i$ th layer. The activation function $f$ often uses the sigmoid function, which is $f(x)=1 /\left(1+e^{-x / \mathrm{T}}\right)$.

In BP neural network, the weight and threshold value of the hidden layer and output layer in BP neural network are adjusted with respective approach. With regard to the output layer,

$$
\begin{aligned}
\Delta \omega_{m n}^{2,3}(k+1) & =\eta \delta_{n} y_{m}^{2}+\alpha \Delta \omega_{m n}^{2,3}(k), \\
\Delta \theta_{n}^{3}(k+1) & =\eta \delta_{n}+\alpha \Delta \theta_{n}^{3}(k), \\
\delta_{n} & =\left(t_{n}-y_{n}^{3}\right) f^{\prime}\left(x_{n}^{3}\right) .
\end{aligned}
$$

To the processing layer, the recurrence formulas are as equations (10)-(12):

$$
\begin{aligned}
\Delta \omega_{i m}^{1,2}(k+1) & =\lambda \delta_{m} y_{i}^{1}+\gamma \Delta \omega_{i m}^{1,2}(k), \\
\Delta \theta_{m}^{2}(k+1) & =\lambda \delta_{m}+\gamma \Delta \theta_{m}^{2}(k), \\
\delta_{m} & =\sum_{n=1}^{d} \delta_{n} \omega_{m n}^{2,3} f^{\prime}\left(x_{m}^{2}\right),
\end{aligned}
$$

where $\lambda$ and $\gamma$ refer to the learning rate, which has a significant impact on the convergence rate and the accuracy of output results, and $\gamma$ represents the momentum coefficient.

The critical issue of the BP neural network topology is to determine the hidden layer number as well as the number of 
neurons, enough of which will markedly improve the network computation ability and exactly approach any complex function of a network with enough hidden layer nodes. However, a large number of hidden layer nodes will result in time-consuming of computation work, and so far, there is no specific formula for determining the hidden layer numbers. According to Zhao [23], in some cases, a relatively few hidden layers can meet the accuracy requirements of actual application, and it is reasonable to take less than twice of the input layer numbers as the hidden layer node numbers.

\subsection{Explicit Formula of Response Function in Dynamic} Coupling Analysis of Vehicle-Bridge System. A typical architecture of a three-layer BP neural network composed of input layer, hidden layer, and output layer (see Figure 2) is employed to derive the explicit formula of response function in dynamic coupling analysis of the vehicle-bridge system, and the bias values and weights in derivations of BP neural network-based formulations are provided in Tables 1 and 2 . The process of a connection weight multiplying each input variable is also conducted.

Suppose that the transpose of the input variable vector is $X^{T}=\left(X_{1}, X_{2}\right)$ and the transpose of the output variable (i.e., response function) vector is $F^{T}=(\hat{F}(X))$ and use the activation function $f(x)=1 /\left(1+e^{-x / T}\right)$ as mentioned above to transfer the input layer node values to the hidden ones, while adopting the linear activation function $F(x)=x$ to transfer the hidden layer node values to the output ones. For the typical architecture of a three-layer BP neural network (see Figure 2), the explicit formula of response function in dynamic coupling analysis of vehicle-bridge system can be written as equation (13), which is

$$
\hat{F}\left(X^{\text {scaled }}\right)=G_{1} \cdot w_{2}(1,1)+G_{2} \cdot w_{2}(1,2)+G_{3} \cdot w_{2}(1,3)+b_{2},
$$

TABLe 1: Weight and bias values between input and processing layers.

\begin{tabular}{lccc}
\hline \multirow{2}{*}{ Weights } & \multicolumn{3}{c}{ Number of processing layer nodes $(i)$} \\
& 1 & 2 & 3 \\
\hline$w_{1 i}$ & $w_{1}(1,1)$ & $w_{1}(1,2)$ & $w_{1}(1,3)$ \\
$w_{2 i}$ & $w_{1}(2,1)$ & $w_{1}(2,2)$ & $w_{1}(2,3)$ \\
Bias & $b_{1}(1,1)$ & $b_{1}(2,1)$ & $b_{1}(3,1)$ \\
\hline
\end{tabular}

where

TABLE 2: Weight values between output and processing layers.

\begin{tabular}{lccc}
\hline \multirow{2}{*}{ Weights } & \multicolumn{3}{c}{ Number of processing layer nodes $(i)$} \\
& 1 & 2 & 3 \\
\hline$w_{1 i}$ & $w_{2}(1,1)$ & $w_{2}(1,2)$ & $w_{2}(1,3)$ \\
Bias & $b_{2}$ & - & - \\
\hline
\end{tabular}

$$
\begin{aligned}
& G_{1}=\frac{1}{1+\mathrm{e}^{-\left(w_{1}(1,1) \cdot X_{1}^{\text {scaled }}+w_{1}(2,1) \cdot X_{2}^{\text {scaled }}+b_{1}(1,1)\right)},} \\
& G_{2}=\frac{1}{1+\mathrm{e}^{-\left(w_{1}(1,2) \cdot X_{1}^{\text {scaled }}+w_{1}(2,2) \cdot X_{2}^{\text {scaled }}+b_{1}(2,1)\right)}}, \\
& G_{3}=\frac{1}{1+\mathrm{e}^{-\left(w_{1}\left((1,3) \cdot X_{1}^{\text {scaled }}+w_{1}(2,3) \cdot X_{2}^{\text {scaled }}+b_{1}(3,1)\right)\right.}} .
\end{aligned}
$$

It should be noted that the explicit formula of response function in dynamic coupling analysis of vehicle-bridge system obtained according to equation (13) was shown as follows:

$$
\hat{F}(X)=\frac{w_{2}(1,1)}{1+\mathrm{e}^{-\left(w_{1}(1,1) \cdot X_{1}+w_{1}(2,1) \cdot X_{2}+b_{1}(1,1)\right)}}+\frac{w_{2}(1,2)}{1+\mathrm{e}^{-\left(w_{1}(1,2) \cdot X_{1}+w_{1}(2,2) \cdot X_{2}+b_{1}(2,1)\right)}}+\frac{w_{2}(1,3)}{1+\mathrm{e}^{-\left(w_{1}(1,3) \cdot X_{1}+w_{1}(2,3) \cdot X_{2}+b_{1}(3,1)\right)}}+b_{2}
$$

where $X$ was the variable while $X^{\text {scaled }}$ was the scaled value of $X$.

\subsection{The Process of BP Neural Network Fitting Dynamic} Coupling Response of Vehicle-Bridge System Response. The general process of applying BP neural network technology to fit the dynamic coupling response of the vehicle-bridge system is as follows:

(1) Enter the corresponding sample points according to the characteristics of the input parameters, in which the longitudinal distribution function along the bridge is obtained on the basis of the power spectral density function of pavement roughness and pavement grade.
(2) Establish the finite element model of bridge structure, calculate the structural response at the sample points, and then constitute the training sample by structure response and sample points obtained through steps (1) and (2), respectively.

(3) Build the BP neural network model to study and optimize the training samples from step (2), and then get the weights and thresholds of the network. Firstly, use the uniform design method to choose suitable training datasets, then determine the architecture of the BP neural network model and backpropagation training algorithm, and after that train the neural network with the selected training datasets. 
(4) Obtain the dynamic response expression of the bridge in the vehicle-bridge coupling system according to weights and thresholds from step (3).

\section{Numerical Example}

A numerical example is given to illustrate the feasibility and applicability of the proposed method. A simply supported girder bridge with a span of $32 \mathrm{~m}$ is considered; the mass per unit length is $5.41 \times 10^{3} \mathrm{~kg} / \mathrm{m}$, and the bending rigidity is $3.5 \times 10^{10} \mathrm{~N} / \mathrm{m}^{2}$. The system stiffness is $6.5 \times 10^{5} \mathrm{~N} / \mathrm{m}$, the system damping is $2.1 \times 10^{4} \mathrm{~N} \cdot \mathrm{s} / \mathrm{m}$, and the system mass is $32025 \mathrm{~kg}$. Assume that the roughness of road surface obeys the Gaussian random process with zero mean, stationarity, and ergodicity, the longitudinal distribution function of which along the bridge is obtained by the power spectral density function of the road surface roughness and the road surface condition. A three-axle vehicle is adopted to drive in one direction at a speed of $60 \mathrm{~km} / \mathrm{h}$, and the whole analysis process starts with the front axle entering into the bridge and ends with the rear axle leaving the bridge. The time interval of numerical simulation is 0.2 seconds. The transient dynamic analysis method is used to obtain the finite element time-history response of the vertical displacement of the midspan joint, which is shown in Figure 4.

Simultaneously, the BP neural network fitting method with a three-layer BP neural network proposed in this paper is adopted to analyze the same numerical example as above. From the simulation results, there are several points, including $0.2 \mathrm{~s}$ and $1.2 \mathrm{~s}$, showing a little degree of deviation and dispersion, but from the perspective of root mean square error and correlation index, which shows a high degree of consistency. In view of the maximum value considered in practical engineering, the relative error is very small that is within the acceptable range of engineering application. As can be seen from Figure 3(b), the bridge dynamic response in the vehicle-bridge coupling system calculated by the BP neural network technology proposed in this paper is in good agreement with the results of the finite element method, which indicates that the dynamic coupling analysis results of vehicle-bridge coupling system conducted by the BP neural network method are accurate and reliable, and the calculation accuracy using BP neural network method can meet the engineering requirements.

\section{Application}

In this section, the BP neural network approach defined in previous sections and the finite element method for dynamic coupling analysis of vehicle-bridge system are applied to an actual suspension bridge, whose span arrangement is $(658+1688+658) \mathrm{m}$, and the overall elevation is shown in Figure 5; more detailed information can be found in the literature [24].

4.1. Finite Element Method. In order to validate the accuracy and applicability of the approach proposed in this paper in solving engineering problems, the finite element analysis is firstly carried out by ANSYS in which the beam element
(Beam44) is adopted to build the stiffening girder and the bridge tower, and the only-tension link element Link10 is to establish the main cable and the hanger. The clamped boundary is applied to the main cable ends as well as the bottom of the bridge tower, and the simply supported boundary is to the stiffening girder. The stiffening girder is discretized according to the lifting point of the suspender. The main girder and lifting point are connected by a rigid element. The stress mode of the main cable unit is set as tension only, not compression. When the bridge is completed, the initial stress of the main cable and hanger is considered as the initial strain of the element. In order to consider the influence of geometric nonlinearity, the elastic modulus of the main cable is calculated by the equivalent Ernst method. The reasonably finished dead state analysis of the suspension bridge is conducted so as to ensure the accuracy of the finite element model. There are 1101 nodes and 2076 elements in the finite element model. The finite element model diagram of the whole suspension bridge is shown in Figure 6.

The analysis model is established on the condition of good flatness of the road surface, and the road roughness function and road roughness derivative in longitudinal direction of the stiffened girder are thus generated, respectively, which is in Figures 7 and 8. In Figure 7, the horizontal ordinate is the longitudinal direction of the stiffened girder and the vertical coordinate is the road roughness, while in Figure 8, the horizontal ordinate is the longitudinal direction of the stiffened girder and the vertical coordinate is the road roughness derivative.

The vehicle load consists of two groups of cars, each of which is constituted by a $30 \mathrm{t}$ three-axis car and a $20 \mathrm{t}$ two-axis car, and each vehicle group travels at the speed of $100 \mathrm{~km} / \mathrm{h}$ from both ends of the bridge at the same time with opposite direction, while the two cars include a $30 \mathrm{t}$ three-axis car and a $20 t$ two-axis car at each group traveling in the same direction in the meantime. Each vehicle group is driven on the right side, and the eccentricity between the two vehicles in each group and the centerline of the bridge deck is $11.75 \mathrm{~m}$ and $8.25 \mathrm{~m}$, respectively. The vehicle, moving in one direction, starts traveling with the side span end of the stiffening beam and ends with traveling out of the stiffening beam, and the whole driving time is $104.54 \mathrm{~s}$.

In order to consider the accuracy and efficiency of data acquisition at the same time, the time interval of $0.2 \mathrm{~s}$ is appropriate [25]. The finite element transient dynamic analysis is carried out with a time step of $0.2 \mathrm{~s}$, and the analysis results of vertical displacement, velocity, and acceleration for midspan nodes are extracted as in Figure 9.

From Figure 9, it can be seen that the vertical displacement, vertical speed, and vertical acceleration of midspan node almost simultaneously reached the maximum value. The maximum value of the vertical displacement and vertical acceleration of midspan node was in $52.27 \mathrm{~s}$, while the maximum value of vertical speed was in $48.81 \mathrm{~s}$ and 58.36 s. So, we can conclude that the maximum response of midspan nodes is near the middle of the history time in order to analyze the vehicle-bridge response in actual engineering application. 


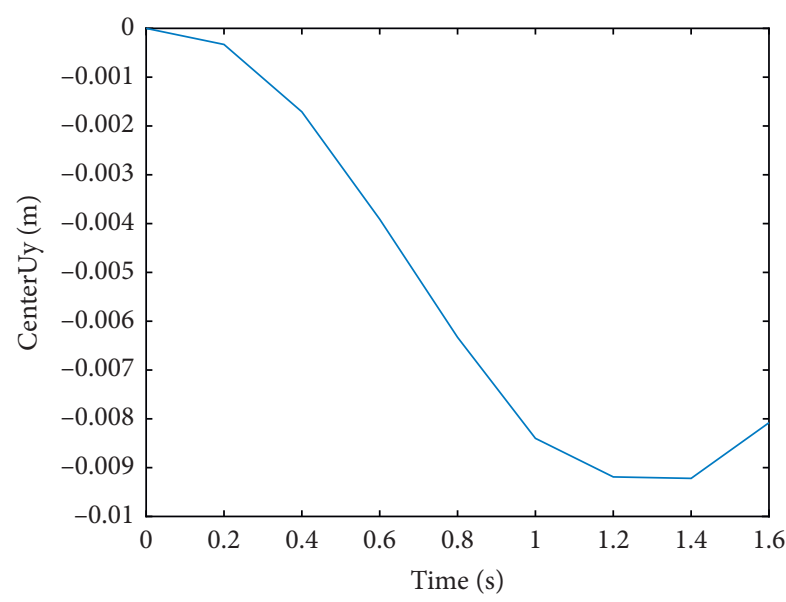

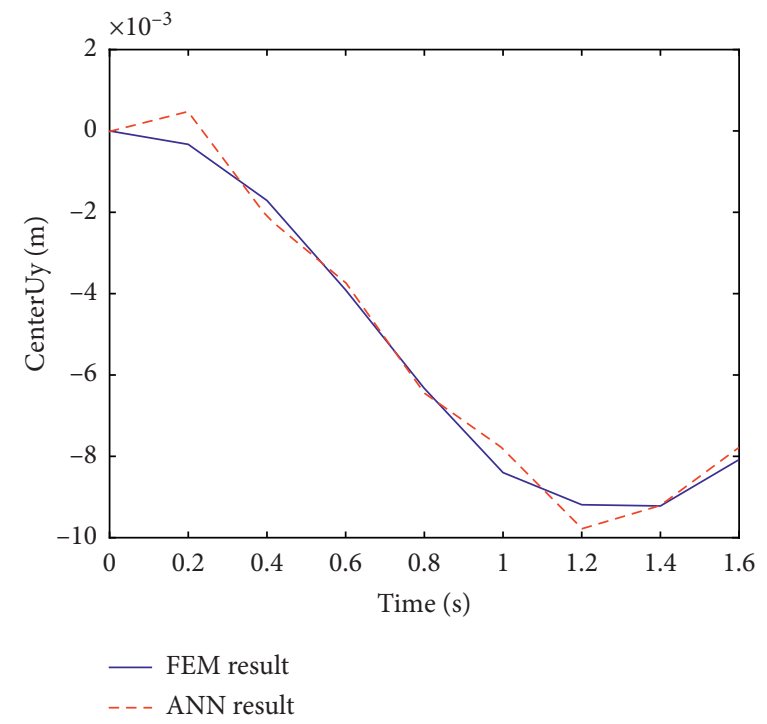

(b)

Figure 4: Time-history response of the vertical displacement of the midspan joint; (a) finite element time-history response of the numerical example; (b) comparison between BP neural network time-history results and finite element time-history results.

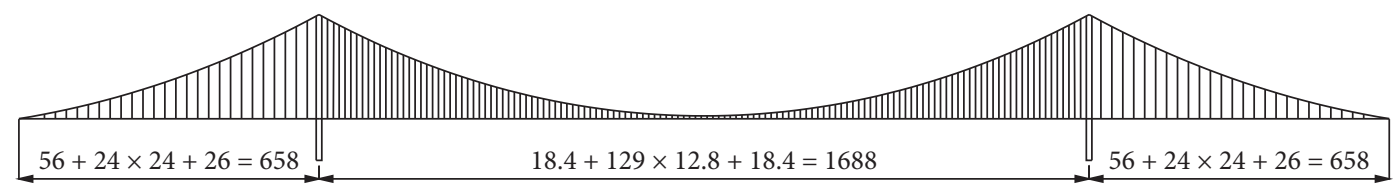

FIgURE 5: Overall elevation of the suspension bridge.

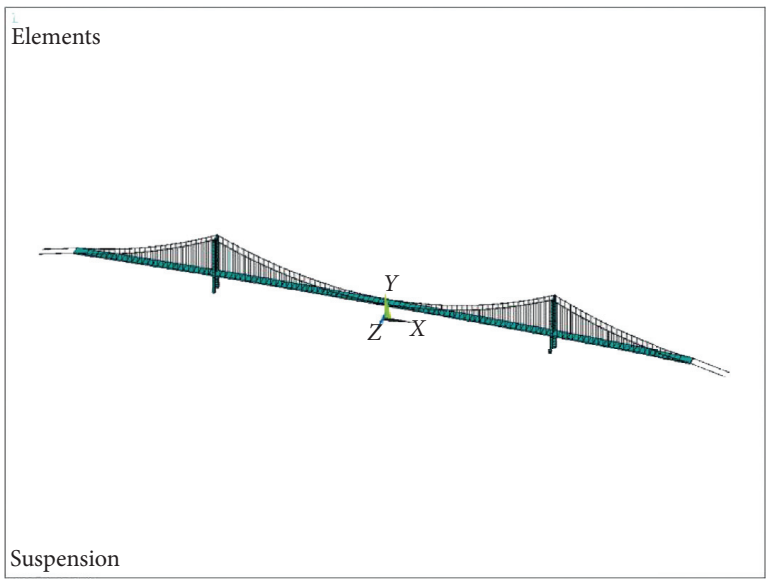

FIGURE 6: Finite element model diagram of the whole suspension bridge.

4.2. BP Neural Network Fitting. The BP neural network approach proposed in this paper is used for fitting approximation of the transient dynamic response of the suspension bridge mentioned before in the vehicle-bridge coupling system, and the weight and the threshold of threelayer BP neural network are obtained accordingly. In order

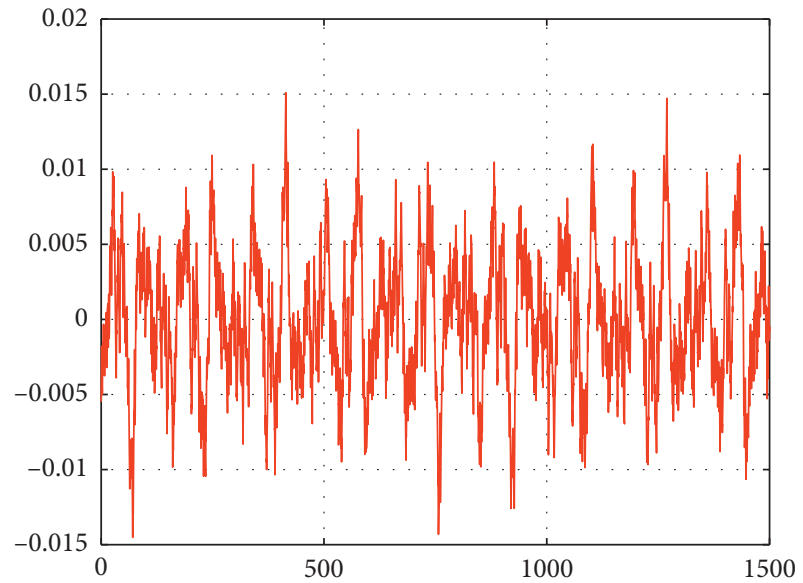

FIGURE 7: Road roughness function in longitudinal direction of the stiffened girder (showing the road roughness and the road roughness derivative of half span length only, the other half is taken symmetrically).

to compare the results analyzed through finite element method and BP neural network approach, the vertical displacement, velocity, and acceleration in the middle of the span are correspondingly chosen for time histories analysis, and the results of the finite element (FEM) and BP neural network approach are drawn in Figure 10. 


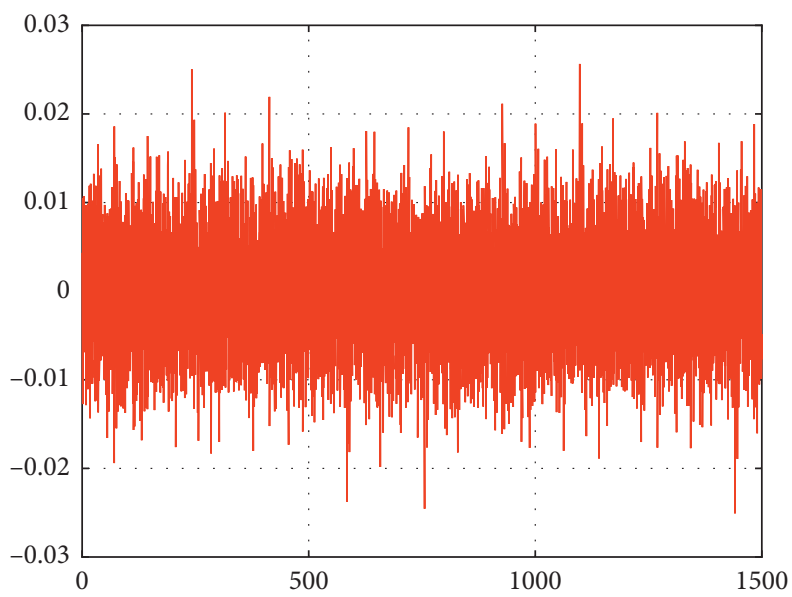

Figure 8: Road roughness derivative in longitudinal direction of the stiffened girder (showing the road roughness and the road roughness derivative of half span length only, the other half is taken symmetrically).

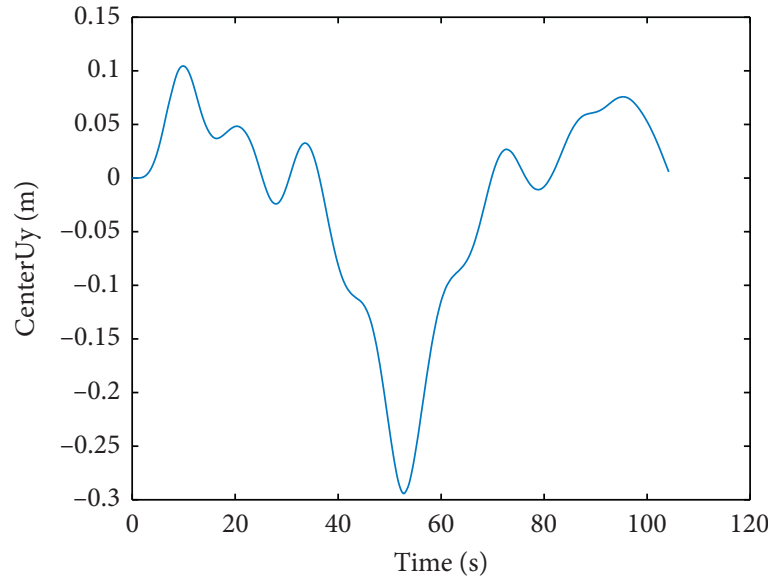

(a)

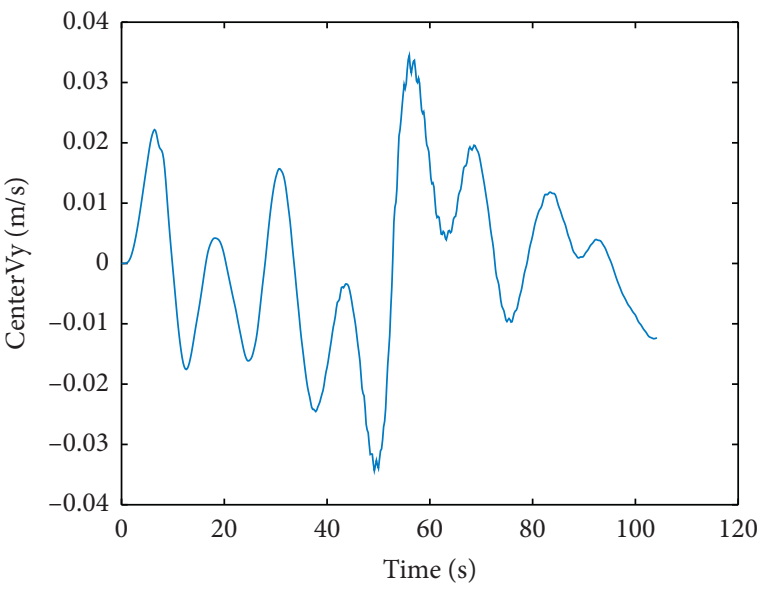

(b)

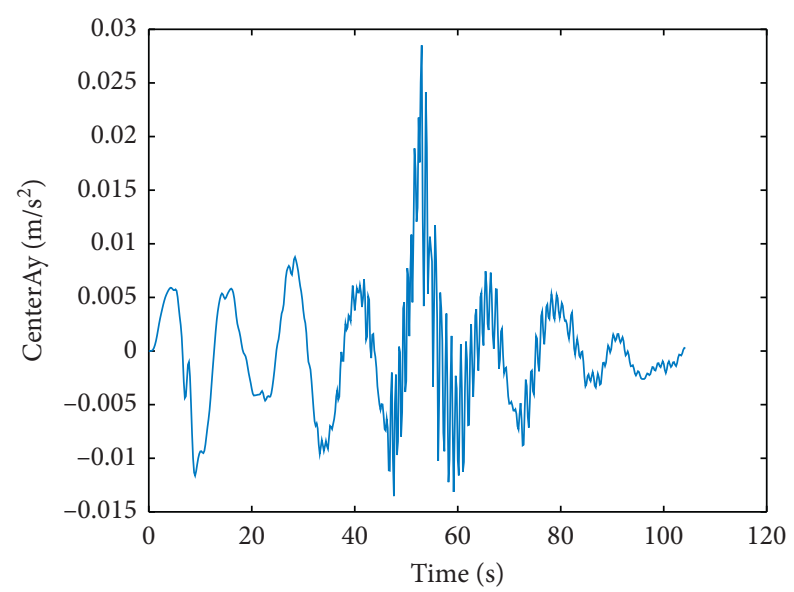

(c)

Figure 9: The finite element transient dynamic analysis results: (a) time-history results of vertical displacement in the middle of the span for dynamic coupling analysis of vehicle-bridge system; (b) time-history results of vertical speed in the middle of the span for dynamic coupling analysis of vehicle-bridge system; (c) time-history results of vertical acceleration in the middle of the span for dynamic coupling analysis of the vehicle-bridge system. 


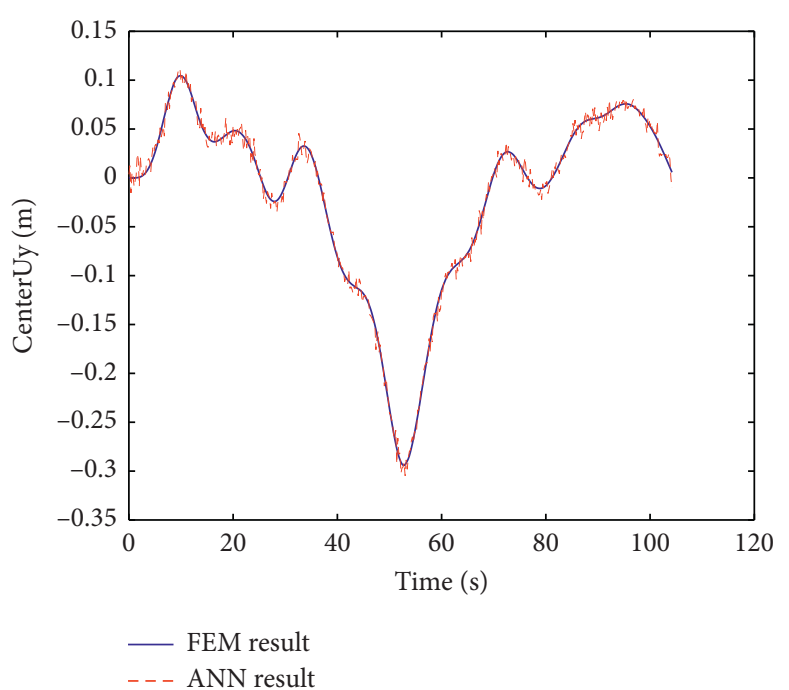

(a)

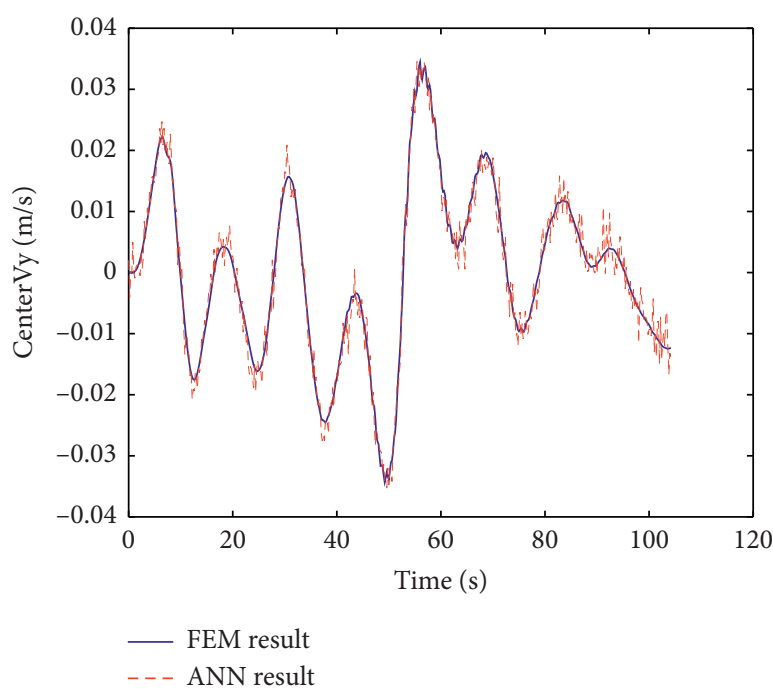

(b)

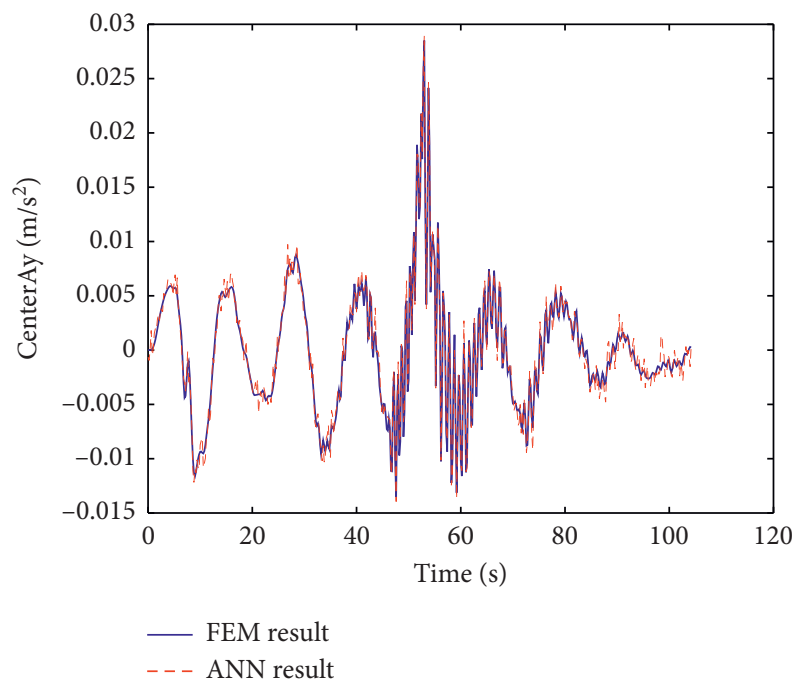

(c)

FIGURE 10: Comparison between BP neural network time-history results and finite element time-history results: (a) comparison of timehistory results of vertical displacement in the middle of the span for dynamic coupling analysis of vehicle-bridge system; (b) comparison of time-history results of vertical speed in the middle of the span for dynamic coupling analysis of vehicle-bridge system; (c) comparison of time-history results of vertical acceleration in the middle of the span for dynamic coupling analysis of the vehicle-bridge system.

Through the detailed analysis of Figure 10, it can be seen that the overall trend between the finite element simulation and the neural network is consistent. However, through detailed analysis, it can be seen that the difference between the finite element simulation and neural network fitting is slightly larger, of which the correlation index is 0.963 between BP neural network fitting and finite element results, in the region with relatively small response value, while the difference is small near the peak value, of which the correlation index is 0.999 between BP neural network fitting and finite element results. The main reason is that the neural network fitting has a little degree of discreteness and difference, which is obvious near the small value, but not obvious near the large value. From the engineering point of view, the main concern is the maximum value of response to control the most unfavorable conditions, so the difference near the maximum value is very small, which shows the applicability of this method in engineering.

According to the comparison results between the $\mathrm{BP}$ neural network approach and finite element method in Figure 10, it is shown that the results of dynamic coupling analysis of the vehicle-bridge system of long-span suspension bridge calculated based on BP neural network are in good agreement with finite element method, which indicates that the BP neural network approach exhibits a better performance in terms of accuracy and feasibility, as measured by the closeness of the exact values calculated by ANSYS software and by CPU running time comparison of the two approaches for dynamic coupling analysis of vehicle- 
TABLE 3: Comparison results of reliability index of vehicle-bridge coupling.

\begin{tabular}{lccc}
\hline Method & Reliability index & Iterative times & Computing time of CPU \\
\hline BP neural network & 8.9218 & 6 & $2 \mathrm{~s}$ \\
Finite element method & 8.9177 & 15 & $3.53 \mathrm{~h}$ \\
\hline
\end{tabular}

bridge system (CUP running time of ANSYS is about $5400 \mathrm{~s}$, while it is $150 \mathrm{~s}$ for the proposed method).

4.3. Reliability Analysis of Driving Comfort. The stiffness of long-span suspension bridge is small, and the vehicle-bridge coupling vibration response under the action of vehicles will affect the driving comfort, so it is paid attention to in practical engineering. In general, the comfort of vehicles in the process of driving is generally measured by the vertical acceleration index. The reliability method has been widely used in the evaluation of driving comfort. The vehicle-bridge coupling vibration response and its gradient are two important problems in reliability calculation, respectively. For suspension bridges, the vehicle-bridge coupling response is an implicit function of basic variables, and the response gradient is difficult to express explicitly. The combination of finite element method and difference method can calculate the response gradient, but it needs to call the finite element many times, so it takes a lot of time. Now, neural network fitting technology and finite element difference method are used to calculate the reliability index of driving comfort, respectively.

The limit state function of driving comfort is as follows:

$$
G=a_{0}-a_{\mathrm{v}}
$$

where $a_{0}$ is the acceleration limit that human can bear and $a_{\mathrm{v}}$ is the acceleration response value of vehicle-bridge coupling vibration.

In equation (16), $a_{0}=3.6 \mathrm{~m} / \mathrm{s}^{2}$, while $a_{\mathrm{v}}$ is an implicit function of basic variables. The characteristics of mathematical statistics for all random variables are assumed following normal distribution and the coefficients of variation are 0.05 . Reliability index was calculated using the first-order second-moment reliability method. The comparison results of reliability index of vehicle-bridge coupling calculated by BP neural network and finite element method were shown in Table 3.

From Table 3, we can see that (1) reliability index of vehicle-bridge coupling using BP neural network was almost equal to that of finite element difference method, (2) iterative times of reliability index of vehicle-bridge coupling using BP neural network were less than that of finite element difference method, and (3) computing time of reliability index of vehicle-bridge coupling using BP neural network was less than that of finite element difference method. As mentioned above, due to the vehicle-bridge coupling response which is an implicit function of basic variables, the calculation cost of the reliability index using BP neural network was less than finite element difference method. It is recommended that BP neural network method of vehicle-bridge coupling can be used to the condition of iterative calculation, especially for reliability index in actual engineering application.

\section{Conclusion}

Aiming at the dynamic coupling analysis of the vehiclebridge system of long-span suspension bridge, the BP neural network approach has been developed to perform this paper. A three-layer BP neural network composed of input layer, hidden layer, and output layer was adapted for the dynamic coupling analysis of the vehicle-bridge system of long-span suspension bridge. By reasonably constructing the topological results of the $\mathrm{BP}$ neural network and training the $\mathrm{BP}$ neural network through a certain number of finite element transient analysis results, the corresponding BP neural network can be used to approximate the transient dynamic response of suspension bridges in dynamic coupling analysis of the vehicle-bridge system.

A numerical example was presented to demonstrate the accuracy and efficiency of the proposed method through the comparison between the results of the FEM and BP neural network, which shows that the proposed method exhibited a better performance in terms of accuracy as measured by the closeness of the exact values. Moreover, a specific engineering application case involving detailed structural information of a long-span suspension bridge is also put forward as well as a finite element mode established with ANSYS software for comparison and verification of the proposed method. The results show that they were consistent with each other, and the BP neural network method could improve the computational efficiency proved by total computational time compared to the FEM method, which indicate that the proposed method is applicability and feasibility in actual engineering application.

In the process of finite element simulation, the vehiclebridge coupling system was simulated by single beam mode while not considering the transverse distribution of the vehicles in spatial effects of the bridge deck. The problem about the transverse distribution of the vehicles in spatial effects of the bridge deck would be investigated in the next step phase. Additionally, for long-span suspension, the effect of bridge-vehicle interaction is not as significant as that of short span bridge, and the specific quantitative results comparing the cases of moving load and moving vehicle need further study while the vehicle-bridge coupling problem at specific speed was investigated in this study at the limitation of time and cost.

\section{Data Availability}

The data used to support the findings of this study are available from the corresponding author upon request.

\section{Conflicts of Interest}

The authors declare that they have no conflicts of interest. 


\section{Acknowledgments}

This work presented herein was supported by Shanxi Province Natural Science Foundation (201901D211121) and the Fundamental Research Funds for the Central Universities, CHD (300102210515).

\section{References}

[1] S.-D. Kwon, J.-S. Lee, J.-W. Moon, and M.-Y. Kim, "Dynamic interaction analysis of urban transit maglev vehicle and guideway suspension bridge subjected to gusty wind," Engineering Structures, vol. 30, no. 12, pp. 3445-3456, 2008.

[2] Y. L. Xu, Q. Li, D. J. Wu, and Z. W. Chen, "Stress and acceleration analysis of coupled vehicle and long-span bridge systems using the mode superposition method," Engineering Structures, vol. 32, no. 5, pp. 1356-1368, 2010.

[3] M. Duan, S. Zhang, X. Wang, and F. Dong, "Mechanical behavior in perfobond rib shear connector with UHPC-steel composite structure with coarse aggregate," KSCE Journal of Civil Engineering, vol. 24, no. 4, pp. 1255-1267, 2020.

[4] H. P. Jiang, J. G. Wang, and F. Qian, "Analysis of vehiclebridge coupling vibration of long span suspension bridge," Journal of Hefei University of Technology (Natural Science), vol. 34, no. 1, pp. 114-118, 2011.

[5] H. Q. Li, M. S. Zhao, and Y. J. Chen, "Research on vehiclebridge coupling vibration response of single tower self-anchored suspension bridge," Journal of Chongqing Jiaotong University (Natural Science, vol. 34, no. 3, pp. 1-6, 2015.

[6] N. H. Ding, L. X. Lin, Y. J. Qian et al., "Study on vehicle-bridge coupling vibration of double chain suspension bridge," Journal of Lanzhou Jiaotong University, vol. 29, no. 1, pp. 95-99, 2010.

[7] X. Z. Li, D. J. Liu, Z. B. Jin et al., “Analysis of vehicle-linebridge coupling vibration of long-span railway suspension bridge," Steel Structure, vol. 25, no. 12, pp. 6-12, 2010.

[8] Y. Liu, X. Kong, C. S. Cai et al., "Driving effects of vehicleinduced vibration on long-span suspension bridges," Structural Control \& Health Monitoring, vol. 24, no. 2, Article ID e1873, 2017.

[9] S. Q. Wang, J. Ma, Y. R. Ren et al., "Dynamic interaction analysis on wind-train-bridge system of long-span railway suspension bridge," Journal of Railway Science and Engineering, vol. 14, no. 6, pp. 1243-1250, 2017.

[10] S. C. Ahalt, P. Chen, C.-T. Chou, S. Kuttuva, and T. E. Little, "The neural shell: a neural network simulation tool," Engineering Applications of Artificial Intelligence, vol. 5, no. 3, pp. 183-192, 1992.

[11] I. Flood and N. Kartam, "Neural networks in civil engineering. I: principles and understanding," Journal of Computing in Civil Engineering, vol. 8, no. 2, pp. 131-148, 1994.

[12] A. Y. Dorogov, "Structural synthesis of fast two-layer neural networks," Cybernetics and Systems Analysis, vol. 36, no. 4, pp. 512-519, 2000.

[13] M. N. S. Hadi, "Neural networks applications in concrete structures," Computers \& Structures, vol. 81, no. 6, pp. 373-381, 2003.

[14] B. B. Adhikary and H. Mutsuyoshi, "Artificial neural networks for the prediction of shear capacity of steel plate strengthened RC beams," Construction and Building Materials, vol. 18, no. 6, pp. 409-417, 2004.

[15] S. H. Ling, F. H. F. Leung, and H. K. Lam, "An improved genetic algorithm based fuzzy-tuned neural network,"
International Journal of Neural Systems, vol. 15, no. 06, pp. 457-474, 2005.

[16] A. H. Elhewy, E. Mesbahi, and Y. Pu, "Reliability analysis of structures using a neural network method," Probabilistic Engineering Mechanics, vol. 21, no. 1, pp. 44-53, 2006.

[17] S. Effati and M. Jafarzadeh, "Nonlinear neural networks for solving the shortest path problem," Applied Mathematics and Computation, vol. 189, no. 1, pp. 567-574, 2007.

[18] J. Cheng, Q. S. Li, and R.-C. Xiao, "A new artificial neural network-based response surface method for structural reliability analysis," Probabilistic Engineering Mechanics, vol. 23, no. 1, pp. 51-63, 2008.

[19] Y. Lv, T. Hu, G. Wang, and Z. Wan, "A neural network approach for solving nonlinear bilevel programming problem," Computers \& Mathematics with Applications, vol. 55, no. 12 , pp. $2823-2829,2008$.

[20] M. Chen, S. S. Ge, and B. V. E. How, "Robust adaptive neural network control for a class of uncertain MIMO nonlinear systems with input nonlinearities," IEEE Transaction on Neural Networks, vol. 21, no. 5, pp. 796-812, 2010.

[21] L. Magnier and F. Haghighat, "Multiobjective optimization of building design using TRNSYS simulations, genetic algorithm, and Artificial Neural Network," Building and Environment, vol. 45, no. 3, pp. 739-746, 2010.

[22] Q. Liu, J. Cao, and G. Chen, "A novel recurrent neural network with finite-time convergence for linear programming," Neural Computation, vol. 22, no. 11, pp. 2962-2978, 2010.

[23] L. M. Zhao and D. H. Wei, "The method to choose the optimal number of hide nodes of artificial neural networks," Journal of North China Institute of Water Conservancy and Hydroelectric Power, vol. 20, no. 4, pp. 44-48, 1999.

[24] X. M. Zhang, Y. Q. Xu, L. T. Lu et al., "Study on longitudinal constraint system of nizhou waterway bridge of second humen bridge," Bridge Construction, vol. 49, no. 2, pp. 10-15, 2019.

[25] D. J. Wu, Q. Li, and A. R. Chen, "Numerical stability analysis of vehicle bridge coupled vibration by iterative method," Chinese Quarterly of Mechanics, vol. 28, pp. 405-411, 2007. 